\title{
Momentum anisotropies in the quark coalescence model
}

\author{
Peter F. Kolb \\ Physik Department, Technische Universität München, D-85747 Garching, Germany \\ Lie-Wen Chen, Vincenzo Greco, and Che Ming Ko \\ Cyclotron Institute and Physics Department, Texas A\&M University, College Station, Texas 77843, USA
}

(Received 17 February 2004; published 21 May 2004)

\begin{abstract}
Based on the quark coalescence model, we derive relations among the momentum anisotropies of mesons and baryons in relativistic heavy ion collisions from a given, but arbitrary azimuthal distribution for the partons. These relations allow us to estimate the higher-order anisotropies in the initial partonic matter from the measured hadronic anisotropies. Besides the familiar even Fourier coefficients such as the elliptic flow, we also pay attention to odd Fourier coefficients such as the directed flow, which has been observed at finite rapidity even at Relativistic Heavy Ion Collider energies.
\end{abstract}

DOI: 10.1103/PhysRevC.69.051901

PACS number(s): 25.75.Ld, 24.10.Lx

The systematic analysis of anisotropies of particle momentum distributions in the plane perpendicular to the beam direction in noncentral collisions of heavy ions offers the possibility to understand both the dynamics of the collisions and the properties of the initially produced hot and dense matter [1]. In the low transverse momentum region, this momentum anisotropy is a result of the hydrodynamic expansion that is driven by anisotropic pressure gradients in the system [2,3]. Particles of intermediate to high energies do not follow this collective dynamics, but instead radiate more or less energy, depending on how much matter they traverse on their way out of the fireball. As a result, these particles also acquire a strong azimuthal anisotropy in their momentum distributions [4]. For heavy ion collisions at the Relativistic Heavy Ion Collider (RHIC), it has been shown in hydrodynamical models [5-8] that the lower-order momentum anisotropy, such as the elliptic flow, is sensitive to the equation of state of the quark-gluon plasma formed during the initial stage of the collision as well as the transition region to hadronic matter in the later stages. In transport models [9-11], the hadron elliptic flow is shown to depend on the parton scattering cross sections in the initial partonic matter. For hadrons with high transverse momentum, the elliptic anisotropy can further provide information on the energy density of the initial hot matter [12].

Although the hadron anisotropies become smaller with increasing order, they seem to be sensitive to the initial collision dynamics as well [13-15]. Recent experimental results from the STAR Collaboration [16] have demonstrated that some of the higher-order harmonics are still experimentally accessible. The experimental data further indicate that there is a scaling relation among the hadronic anisotropy coefficients $v_{n}$, i.e., $v_{n}\left(p_{T}\right) \sim 1.2 v_{2}^{n / 2}\left(p_{T}\right)$. To gain insight in the origin of this scaling relation, we relate in the present paper the hadron anisotropies to those of quarks using the quark coalescence model. In particular, a naive quark coalescence model $[17,18]$, which ignores the momentum distribution of quarks inside hadrons, will be used. Recently, this model has been shown to give a natural explanation for another scaling law observed in experiments: the momentum dependence of the elliptic anisotropy of identified particles (with the excep- tion of pions) becomes similar if both anisotropy and momentum are divided by the number of constituent quarks in the hadron, i.e., two for mesons and three for baryons. In Ref. [19], it was shown that the deviation of the pion elliptic flow from the scaling law could be explained by taking into account the effect of resonance decays as well as the momentum distribution of quarks in hadrons as in the more realistic quark coalescence model [20-22].

The azimuthal distribution of the particle transverse momentum spectra (or the probability of having a particle with a given transverse momentum $p_{T}$ and azimuthal angle $\varphi$ ) can in general be expressed in terms of the Fourier series

$$
f\left(\varphi, p_{T}\right) \propto 1+2 \sum_{n=1}^{\infty} v_{n}\left(p_{T}\right) \cos n \varphi,
$$

where $v_{n}$ denotes the $n$th order momentum anisotropy. In the above, we have chosen the beam direction for the $z$ axis and the impact parameter for the $x$ axis. Sine terms which are in general possible in a Fourier expansion always vanish in these coordinates because of the reflection symmetry with respect to the reaction plane [the $(x, z)$ plane]. For collisions of equal nuclei, the odd Fourier coefficients vanish at midrapidity due to the additional symmetry with respect to the $y$ axis. This symmetry is lost at forward and backward rapidities as a result of spectator matter from the initial nuclei, which eventually leads to a tilt of the reaction zone out of the transverse plane. Collisions of nonidentical nuclei such as $\mathrm{S}+\mathrm{Au}$ break this symmetry even at midrapidity. It is therefore of great interest to keep the odd coefficients in our general considerations.

In the simplest coalescence model [17], mesons of transverse momentum $p_{T}$ are formed by quarks and antiquarks of half the transverse momentum $p_{T} / 2$ traveling in the same direction. Since the azimuthal distribution function, Eq. (1), after normalizing appropriately, can be interpreted as a probability distribution in the azimuthal angle, the distribution function for mesons is given by the square of that for partons, and it can also be expressed in terms of a Fourier series, i.e., 


$$
F(\varphi) \propto f(\varphi)^{2} \propto 1+2 \sum_{n=1}^{\infty} V_{n} \cos n \varphi,
$$

where $f(\varphi)$ given by Eq. (1) is taken to be the azimuthal distribution of the parton transverse momentum spectra, and $V_{n}$ denotes the $n$th order meson anisotropy. Following the calculations outlined in the Appendix, we arrive at the following expression for $V_{n}$ at momentum $p_{T}$ in terms of the parton anisotropy $v_{n}$ at momentum $p_{T} / 2$ :

$$
V_{n}=\frac{1}{N}\left(2 v_{n}+\sum_{i=1}^{n-1} v_{i} v_{n-i}+2 \sum_{i=1}^{\infty} v_{i} v_{n+i}\right),
$$

with

$$
N=1+2 \sum_{i=1}^{\infty} v_{i}^{2}
$$

Similarly, we obtain the Fourier spectra of baryons by taking the azimuthal distribution of the parton transverse momentum spectra to the third power, i.e.,

$$
\tilde{F}(\varphi) \propto f(\varphi)^{3} \propto 1+2 \sum_{n=1}^{\infty} \tilde{V}_{n} \cos n \varphi .
$$

The calculations given in the Appendix then lead to a somewhat more complicated expression for the $n$th order baryon anisotropy $\tilde{V}_{n}$,

$$
\begin{aligned}
\tilde{V}_{n}= & \frac{1}{\tilde{N}}\left(3 v_{n}+3 \sum_{i=1}^{n-1} v_{i} v_{n-i}+6 \sum_{i=1}^{\infty} v_{i} v_{n+i}+3 \sum_{i=1}^{\infty} \sum_{j=1}^{\infty} v_{i} v_{j} v_{n+i+j}\right. \\
& \left.+3 \sum_{i=1}^{\infty} \sum_{j=1}^{n+i-1} v_{i} v_{j} v_{n+i-j}+\sum_{i=1}^{n-1} \sum_{j=1}^{n-i-1} v_{i} v_{j} v_{n-(i+j)}\right)
\end{aligned}
$$

with

$$
\tilde{N}=1+6 \sum_{i=1}^{\infty} v_{i}^{2}+6 \sum_{i=1}^{\infty} \sum_{j=1}^{\infty} v_{i} v_{j} v_{i+j}
$$

In the above, the parton anisotropy $v_{n}$ 's on the right hand side of the equation are determined at a momentum that is $1 / 3$ of the baryon momentum at which the baryon anisotropy $\tilde{V}_{n}$ 's on the left side of the equation are evaluated.

Dominant and clearly visible in the expressions above is the linear connection between the $n$th harmonic of the partonic sector and the $n$th harmonic of the hadron sector, scaled by a factor 2 and 3 for mesons and baryons, respectively. Corrections to these linear relations are at least of the order $v_{n}^{2}$ and should therefore be small. This gives us a first hint that if the experimental data exhibit a strong $v_{4}$ component in the particle momentum anisotropy as was predicted in Refs. $[15,23]$ and recently confirmed by the STAR Collaboration [16], it is then a true physical component in the transverse distribution, and not just an artifact or trivial byproduct of the existing large $V_{2}$ signal. We will return to this discussion repeatedly.
We now proceed to study Eqs. (3) and (6) under the assumption that higher-order coefficients are small corrections in the distribution and can thus be neglected from the sums at certain order in $n$.

In the first two years of RHIC physics, a vast amount of data from $\mathrm{Au}+\mathrm{Au}$ collisions were collected primarily at midrapidity. Due to the symmetry of those systems, the odd Fourier coefficients in the expansion of the azimuthal distribution of particle transverse momentum vanish both on the partonic and hadronic sector.

Meson anisotropy at midrapidity. Keeping terms up to the order $v_{6}$ and neglecting higher-order corrections, we find from Eq. (3) that for mesons (up to order $V_{6}$ )

$$
\begin{gathered}
V_{2}=\frac{1}{N}\left(2 v_{2}+2 v_{2} v_{4}+2 v_{4} v_{6}\right), \\
V_{4}=\frac{1}{N}\left(2 v_{4}+v_{2}^{2}+2 v_{2} v_{6}\right),
\end{gathered}
$$

$$
V_{6}=\frac{1}{N}\left(2 v_{6}+2 v_{2} v_{4}\right),
$$

with $N=1+2\left(v_{2}^{2}+v_{4}^{2}+v_{6}^{2}\right)$.

Interestingly, we notice that $V_{4}$ of the mesons can be generated out of the partonic sector even if the partonic distribution has only an elliptic component $v_{2}$, but no $v_{4}$ contribution. This is an effect of the nonlinear way how coalescence fuses partons into hadrons [i.e., by squaring or cubing the quark distribution function, see Eqs. (2) and (5)]. The resulting $V_{4}$ is however small, namely, for a maximum parton anisotropy $v_{2}$ of about $10 \%$ as required to describe the experimental hadron anisotropy one obtains $V_{4} \sim 1 \%$.

Neglecting terms including $v_{6}$ in the equations above we find

$$
\frac{V_{4}}{V_{2}^{2}} \approx \frac{1}{4}+\frac{1}{2} \frac{v_{4}}{v_{2}^{2}}
$$

Experimentally [16], this ratio (measured for all charged hadrons) is approximately constant as a function of transverse momentum, and has a value of about 1.2. Therefore, we can be quite certain that there are higher-order harmonics in the Fourier expansion of the azimuthal dependence of the parton transverse momentum distribution. In fact we can deduce from this observation that $v_{4}\left(p_{T}\right) \sim 2 v_{2}^{2}\left(p_{T}\right)$. A finite parton $v_{4}$, although it scales with $v_{2}^{2}$ with a scaling factor of only about 1 , is indeed seen in the predicted parton anisotropies [23] from the AMPT (A MultiPhase Transport) model [24], which is based on a coalescence picture that combines two nearest quarks and antiquarks into a meson and three nearest quarks or antiquarks into a baryon or an antibaryon that is close to the invariant mass of these partons.

Baryon anisotropy at midrapidity. For the Fourier coefficients of the baryon anisotropy, we again consider coefficients up to sixth order. From Eq. (6), we obtain 


$$
\begin{aligned}
\tilde{V}_{2}= & \frac{1}{\tilde{N}}\left(3 v_{2}+6 v_{2} v_{4}+6 v_{4} v_{6}+3 v_{2}^{3}+3 v_{2}^{2} v_{6}+6 v_{2} v_{4}^{2}+6 v_{2} v_{6}^{2}\right. \\
& \left.+3 v_{4}^{2} v_{6}\right) \\
\tilde{V}_{4}= & \frac{1}{\tilde{N}}\left(3 v_{4}+3 v_{2}^{2}+6 v_{2} v_{6}+6 v_{2}^{2} v_{4}+6 v_{2} v_{4} v_{6}+3 v_{4}^{3}+6 v_{4} v_{6}^{2}\right)
\end{aligned}
$$

$$
\tilde{V}_{6}=\frac{1}{\tilde{N}}\left(3 v_{6}+6 v_{2} v_{4}+v_{2}^{3}+6 v_{2}^{2} v_{6}+6 v_{4}^{2} v_{6}+v_{2} v_{4}^{2}\right),
$$

with $\tilde{N}=1+6\left(v_{2}^{2}+v_{4}^{2}+v_{6}^{2}\right)+6\left(v_{2}^{2} v_{4}+2 v_{2} v_{4} v_{6}\right)$. We note that to obtain the anisotropy values at momentum $p_{T}$ on the left hand side for baryons, we have to use the parton anisotropy coefficients at $p_{T} / 3$ on the right hand side. As already discussed before, there is a linear connection of quark and baryon anisotropies [17], $\widetilde{V}_{n}\left(p_{T}\right) \approx 3 v_{n}\left(p_{T} / 3\right)$ with corrections which are of the order $v_{n}^{2}$.

Again, we express the ratio of the fourth-order coefficient $\widetilde{V}_{4}$ to the square of the elliptic coefficient $\widetilde{V}_{2}$ up to second order in partonic $v_{n}$, and the result is

$$
\frac{\widetilde{V}_{4}}{\widetilde{V}_{2}^{2}} \approx \frac{1}{3}+\frac{1}{3} \frac{v_{4}}{v_{2}^{2}} .
$$

This confirms the earlier argument that the value observed by the STAR Collaboration can only be achieved if there is a significant $v_{4}$ component on the quark sector. Both equations (11) and (15) show that the anisotropy ratio $v_{4} / v_{2}^{2}$ in the quark sector is larger than the ratio observed in the hadronic sector as soon as the latter is found to be greater than $1 / 2$. Again, we remind the reader that the experimental value is greater than 1 [16], pointing in fact to a large $v_{4}$ component in the partonic sector. It is now up to extensive dynamical studies within different approaches to illuminate the detailed dynamical origin of this component, its relation to the initial conditions of the reaction, the nuclear equation of state, and the possible effects of dissipation.

Together, Eqs. (11) and (15) have the immediate consequence that independent of the detailed centrality or momentum dependence of the quark anisotropies, there is a general relation between baryon and meson anisotropies,

$$
\frac{\widetilde{V}_{4}}{\widetilde{V}_{2}^{2}}\left(3 p_{T}\right) \approx \frac{2}{3} \frac{V_{4}}{V_{2}^{2}}\left(2 p_{T}\right)+\frac{1}{6} .
$$

An experimental investigation of the ratios for identified particles thus proves promising to further approve or disprove the quark coalescence picture, which we leave here as a challenge for future experimental analysis and correspondingly for the coalescence model. Improvements of the model, in particular, accounting for the effects of resonance decays and in-hadron momentum distributions of the partons [19] will clearly distort our given scaling formulas to some degree. Momentum anisotropies of kaons and $\Lambda$ 's, however, are ac- cessible over a broad momentum region by the STAR detector and should not be largely influenced by contributions of decay products.

The symmetry $x \leftrightarrow-x$, which is apparent for collisions of equal nuclei, is broken at nonzero rapidity. This allows for the presence of odd Fourier coefficients both in the partonic and hadronic sectors. This specific symmetry is also lost at midrapidity for collisions of nonequal nuclei, such as $\mathrm{S}$ + Au. Even if one does not expect to create a thermalized system in smaller systems, such as in last year's $d+$ Au run at RHIC, the following discussion is also useful to quantify and relate the different azimuthal coefficients at midrapidity.

Meson anisotropy. We restrict our discussion to the first four coefficients $v_{1}$ to $v_{4}$ in the partonic distribution. It then follows directly from Eq. (3) that

$$
\begin{gathered}
V_{1}=\frac{1}{N_{y}}\left(2 v_{1}+2 v_{1} v_{2}+2 v_{2} v_{3}+2 v_{3} v_{4}\right), \\
V_{2}=\frac{1}{N_{y}}\left(2 v_{2}+v_{1}^{2}+2 v_{1} v_{3}+2 v_{2} v_{4}\right), \\
V_{3}=\frac{1}{N_{y}}\left(2 v_{3}+2 v_{1} v_{2}+2 v_{1} v_{4}\right), \\
V_{4}=\frac{1}{N_{y}}\left(2 v_{4}+v_{2}^{2}+2 v_{1} v_{3}\right),
\end{gathered}
$$

with $N_{y}=1+2\left(v_{1}^{2}+v_{2}^{2}+v_{3}^{2}+v_{4}^{2}\right)$.

As for the even coefficients in the section before, we see that the mesonic $V_{n}$ is linearly related to the partonic $v_{n}$ with corrections that are at least quadratic in $v_{n}$. Even more interesting is that here a meson $V_{2}$ can be generated purely through the partonic directed flow $v_{1}$ without any dipole deformation in the initial matter.

Another interesting point is the relation between harmonics of different orders. As we have already discussed, the experimental data at midrapidity indicate a scaling of $V_{4}$ with $V_{2}^{2}$ as also insinuated by the coalescence model. At forward rapidity, we can investigate a similar relation for $V_{3}$. We notice that a relation between $V_{3}$ and $V_{1}^{2}$ is unlikely because the product of odd harmonics gives rise to an even one, and this is indeed the reason why there are no $v_{2 n+1}^{2}$ in $V_{2 n+1}$ while they are present in $V_{2 n}$. This suggests a possible relation between $V_{3}$ and the odd-even combination $V_{1} V_{2}$. From the above formulas, we find that

$$
\frac{V_{3}}{V_{1} V_{2}} \approx \frac{1}{2}+\frac{1}{2} \frac{v_{3}}{v_{1} v_{2}}+\frac{1}{2} \frac{v_{4}}{v_{2}} \text {. }
$$

The presence of non-negligible odd harmonics at forward rapidity can also reflect on the ratio of fourth to second component by adding a new term to Eq. (11), i.e.,

$$
\frac{V_{4}}{V_{2}^{2}} \approx \frac{1}{4}+\frac{1}{2} \frac{v_{4}}{v_{2}^{2}}+\frac{1}{2} \frac{v_{1} v_{3}}{v_{2}^{2}} .
$$

Baryon anisotropy. The expressions for the baryon anisotropy coefficients are rather lengthy but become easily acces- 
sible when dropping the terms that contain products of three $v_{n}$ coefficients, i.e., by dropping the last three terms of Eq. (6). The resulting approximate expressions are then

$$
\begin{gathered}
\tilde{V}_{1} \approx \frac{1}{\tilde{N}_{y}}\left[3 v_{1}+6\left(v_{1} v_{2}+v_{2} v_{3}+v_{3} v_{4}\right)\right], \\
\tilde{V}_{2} \approx \frac{1}{\tilde{N}_{y}}\left[3 v_{2}+3\left(v_{1}^{2}+2 v_{1} v_{3}+2 v_{2} v_{4}\right)\right], \\
\tilde{V}_{3} \approx \frac{1}{\tilde{N}_{y}}\left[3 v_{3}+3\left(2 v_{1} v_{2}+2 v_{1} v_{4}\right)\right], \\
\tilde{V}_{4} \approx \frac{1}{\tilde{N}_{y}}\left[3 v_{4}+3\left(2 v_{1} v_{3}+v_{2}^{2}\right)\right],
\end{gathered}
$$

and

$$
\tilde{N}_{y} \approx 1+6\left(v_{1}^{2}+v_{2}^{2}+v_{3}^{2}+v_{4}^{2}\right) .
$$

Again, we notice that the $n$th coefficient of the baryon spectra is linearly related to the $n$th coefficient in the partonic sector, and higher-order terms are corrections of the order $v_{n}^{2}$. However, the above expressions also show that even harmonics in the baryon spectra can be generated from only odd coefficients in the quark sector, even in the absence of even partonic harmonics.

As in the case of mesons, it is of interest to look at the ratio between the third and the first two components in the azimuthal distribution of baryon momentum spectra. In the coalescence picture, this is given by

$$
\frac{\tilde{V}_{3}}{\tilde{V}_{1} \tilde{V}_{2}} \approx \frac{2}{3}+\frac{1}{3} \frac{v_{3}}{v_{1} v_{2}}+\frac{2}{3} \frac{v_{4}}{v_{2}} .
$$

This relation as well as that given by Eq. (21) allow us also to investigate the presence of a nonvanishing $v_{3}$ at quark level. In fact, if $v_{3}$ is equal to zero, we have the ratio $V_{3} /\left(V_{1} V_{2}\right) \simeq 0.5$ for mesons and $\tilde{V}_{3} /\left(\tilde{V}_{1} \tilde{V}_{2}\right) \simeq 0.7$ for baryons. Therefore, the existence of a quark $v_{3}$ component can be investigated by looking at the magnitude of this ratio from experimental data, as done for $v_{4}$.

Similar to the case of hadrons at midrapidity in collisions of equal mass nuclei, where baryon $\widetilde{V}_{4} / \widetilde{V}_{2}^{2}$ and meson $V_{4} / V_{2}^{2}$ are related by Eq. (16), we have for the case of nonvanishing odd-order anisotropies the following relation:

$$
\frac{\tilde{V}_{3}}{\tilde{V}_{1} \tilde{V}_{2}}\left(3 p_{T}\right) \approx \frac{2}{3} \frac{V_{3}}{V_{1} V_{2}}\left(2 p_{T}\right)+\frac{1}{3},
$$

if the small $v_{4}$ contribution is neglected in Eqs. (21) and (28).

We want to point out that the above considerations for both mesons and baryons are applicable in a rapidity region where the hadrons are produced by coalescence from the previously established deconfined phase. These formulas therefore do not capture the region of highest rapidity, the fragmentation region, which is located around beam rapidity.
In rapidity, our considerations might therefore be applicable up to $75 \%$ of the beam rapidity, while in transverse momentum space, the pure coalescence picture can be trusted up to $p_{T} \approx 5 \mathrm{GeV}$.

Using a naive quark coalescence model for hadron production from the quark-gluon plasma formed in relativistic heavy ion collisions, which only allows partons of equal momenta to recombine, we have expressed the Fourier coefficients of the azimuthal distribution of meson and baryon momentum spectra in terms of those of partons. Neglecting higher-order terms, simple relations are found between the ratio of the hadrons fourth-order term to the square of their second order to the corresponding ratio for the partons. Furthermore, a simple relation is obtained between such ratios for baryons and mesons, i.e., Eq. (16). As this relation is not further influenced by the detailed structure of the quark distribution, this poses an important prediction of the coalescence model in its region of applicability at intermediate $p_{T}$. Effects of flow and jet quenching that shape the underlying quark distribution do not appear explicitly when comparing the anisotropy ratio of mesons and baryons. For hadrons at finite rapidities or spectra from collisions of nonidentical nuclei, odd-order terms are nonzero. We have shown that the ratio of their third-order term to the product of their firstorder and second-order terms is also simply related to the corresponding ratio for the partons. To account for the observed large ratio $(\sim 1.2)$ of hadron fourth order to the square of second-order anisotropy, a larger value $(\sim 2)$ for the corresponding ratio for the partonic anisotropy is needed. The present analysis based on the schematic quark coalescence model allows one to have a simple understanding of the effect of higher-order partonic anisotropies on those of the observed hadrons.

This work was supported in parts by BMBF and DFG, the U.S. NSF (Grant No. PHY-0098805), the Welch Foundation (Grant No. A-1358), INFN, and the NSF of China (Grant No. 10105008).

\section{APPENDIX}

To derive the harmonic coefficients on the hadronic sector, consider an arbitrary distribution function $f(\varphi)$ on the interval $\varphi \in[0,2 \pi]$ expressed in terms of its Fourier series

$$
f(\varphi)=\sum_{k=0}^{\infty} f_{k} \cos k \varphi .
$$

Squaring this function, as it is required for the meson distributions, we obtain

$$
\begin{aligned}
F(\varphi) & =f(\varphi)^{2}=\sum_{k=0}^{\infty} \sum_{l=0}^{\infty} f_{k} f_{l} \cos k \varphi \cos l \varphi \\
& =\frac{1}{2} \sum_{k=0}^{\infty} \sum_{l=0}^{\infty} f_{k} f_{l}[\cos (k+l) \varphi+\cos (k-l) \varphi] .
\end{aligned}
$$

From this we then find the term independent of the azimuthal angle 


$$
F_{0}=f_{0}^{2}+\sum_{k=0}^{\infty} f_{k}^{2}
$$

and the amplitude of the $n$th harmonic

$$
F_{n}=\frac{1}{2} \sum_{k=0}^{n} f_{k} f_{n-k}+\sum_{k=0}^{\infty} f_{k} f_{n+k}
$$

Substituting $f_{0}=1$ and $f_{k}=2 v_{k}$, we obtain the expressions given in Eq. (3).

For baryons, we have to apply the third power to the function $f(\varphi)$, which leads to

$$
\begin{aligned}
\widetilde{F}(\varphi)= & f(\varphi)^{3}=\sum_{k=0}^{\infty} \sum_{l=0}^{\infty} \sum_{m=0}^{\infty} f_{k} f_{l} f_{m} \cos k \varphi \cos l \varphi \cos m \varphi \\
= & \frac{1}{4} \sum_{k, l, m} f_{k} f_{l} f_{m}[\cos (k+l+m) \varphi+\cos (k+l-m) \varphi \\
& +\cos (k-l+m) \varphi+\cos (k-l-m) \varphi]
\end{aligned}
$$

$$
=\frac{1}{4} \sum_{k, l, m} f_{k} f_{l} f_{m}[\cos (k+l+m) \varphi+3 \cos (k+l-m) \varphi],
$$

where the last equality follows from the repeatedly occurring summation indices in the line above. Again, we can quickly separate the terms with a specific amplitude and find

$$
\widetilde{F}_{0}=\frac{1}{4} f_{0}^{3}+\frac{3}{4} \sum_{k, l}^{\infty} f_{k} f_{l} f_{k+l}
$$

and

$$
\begin{aligned}
\widetilde{F}_{n}= & \frac{3}{4} \sum_{k=0}^{\infty} \sum_{l=0}^{\infty} f_{k} f_{l} f_{n+k+l}+\frac{3}{4} \sum_{k=0}^{\infty} \sum_{l=0}^{n+k} f_{k} f_{l} f_{n+k-l} \\
& +\frac{1}{4} \sum_{k=0}^{n} \sum_{l=0}^{n-k} f_{k} f_{l} f_{n-k-l} .
\end{aligned}
$$

With the substitution $f_{0}=1$ and $f_{k}=2 v_{k}$, one then obtains Eq. (6).
[1] W. Reisdorf and H. G. Ritter, Annu. Rev. Nucl. Part. Sci. 47, 663 (1997).

[2] J.-Y. Ollitrault, Phys. Rev. D 46, 229 (1992).

[3] P. F. Kolb, J. Sollfrank, and U. Heinz, Phys. Rev. C 62, 054909 (2000).

[4] X. N. Wang, Phys. Rev. C 63, 054902 (2001).

[5] D. Teaney, J. Lauret, and E. V. Shuryak, Phys. Rev. Lett. 86, 4783 (2001).

[6] P. F. Kolb, P. Huovinen, U. Heinz, and H. Heiselberg, Phys. Lett. B 500, 232 (2001).

[7] P. Huovinen, P. F. Kolb, U. Heinz, P. V. Ruuskanen, and S. Voloshin, Phys. Lett. B 503, 58 (2001).

[8] P. F. Kolb and U. Heinz, in Quark Gluon Plasma, edited by R. C. Hwa and X. N. Wang (World Scientific, Singapore, 2004) Vol. 3, p. 634.

[9] B. Zhang, M. Gyulassy, and C. M. Ko, Phys. Lett. B 455, 45 (1999).

[10] D. Molnar and M. Gyulassy, Nucl. Phys. A698, 379 (2002).

[11] Z. W. Lin and C. M. Ko, Phys. Rev. C 65, 034904 (2002).

[12] M. Gyulassy, I. Vitev, and X. N. Wang, Phys. Rev. Lett. 86, 2537 (2001).

[13] P. F. Kolb, J. Sollfrank, and U. Heinz, Phys. Lett. B 459, 667 (1999).

[14] D. Teaney and E. V. Shuryak, Phys. Rev. Lett. 83, 4951
(1999).

[15] P. F. Kolb, Phys. Rev. C 68, 031902(R) (2003).

[16] J. Adams et al., STAR Collaboration, Phys. Rev. Lett. 92, 062301 (2004).

[17] D. Molnar and S. A. Voloshin, Phys. Rev. Lett. 91, 092301 (2003).

[18] Z. W. Lin and D. Molnar, Phys. Rev. C 68, 044901 (2003).

[19] V. Greco and C. M. Ko, nucl-th/0402020.

[20] R. J. Fries, B. Müller, C. Nonaka, and S. A. Bass, Phys. Rev. C 68, 044902 (2003); Phys. Rev. Lett. 90, 202303 (2003); C. Nonaka, R. J. Fries, and S. A. Bass, Phys. Lett. B 583, 73 (2004).

[21] V. Greco, C. M. Ko, and P. Levai, Phys. Rev. C 68, 034904 (2003); Phys. Rev. Lett. 90, 202302 (2003).

[22] R. C. Hwa and C. B. Yang, Phys. Rev. C 67, 034902 (2003).

[23] L. W. Chen, C. M. Ko, and Z. W. Lin, Phys. Rev. C 69, 031901(R) (2004).

[24] B. Zhang, C. M. Ko, B. A. Li, and Z. W. Lin, Phys. Rev. C 61, 067901 (2000); Z. W. Lin, S. Pal, C. M. Ko, B. A. Li, and B. Zhang, ibid. 64, 011902 (2001); Nucl. Phys. A698, 375c (2002); Z. W. Lin and C. M. Ko, Phys. Rev. C 65, 034904 (2002); Z. W. Lin, C. M. Ko, and S. Pal, Phys. Rev. Lett. 89, 152301 (2002). 